\title{
Development and preliminary evaluation of the VA Seattle foot
}

\author{
ERNEST M. BURGESS, M.D.; DONALD L. POGGI, A.S.M.E.; DREW A. \\ HITTENBERGER, C.P.; JOSEPH H. ZETTL, C.P.*; DAVID E. MOELLER, I.D.S.A.; \\ KENNETH L. CARPENTER, B.S.; SHIRLEY M. FORSGREN* \\ "Prosthetics Research Study; and \\ + Model and Instrument Works, Inc.; Seattle, Washington
}

\section{INTRODUCTION}

Most mobility aids for physically handicapped individuals seek to restore and improve function that primarily relates to basic lifestyle needs. This is an appropriate priority. With the lower limb amputee, this objective means stable, bipedal standing, and walking on unobstructed level surfaces. These elementary needs should be accomplished with comfort and with reasonable energy output $(7,19)$. Presently available lower limb prostheses effectively satisfy these needs in most instances. However, as the mobility demands of an individual with amputation expand, conventional prostheses in general perform poorly. This circumstance is most evident when the amputee attempts to run. Incremental increases in speed through fast walking, jogging, and running rapidly cause gait alterations in which, with increasing speed, the unilateral amputee spends less and less time and weight on the deficient limb, which results in the sound limb largely propelling the body through the gait cycle. This resulting high-energy consuming, uncomfortable, unstable, and unsightly gait pattern is thus generally avoided. Very few bilateral leg amputees are able to run. For these reasons

This work was supported by Veterans Administration Contract V663P-1667, Rehabilitation Research and Development Funds. most amputees do not walk rapidly or run, and many have never even attempted to do so $(5,6$, $13,14)$.

The ability to move quickly and especially to run is a basic need for most physical recreation. It is also important to physical and mental well being and as a defense against injuries such as falling and avoiding threatening environmental situations.

The Prosthetics Research Unit has investigated in depth over the past seven years the running capabilities of a number of types and levels of lower limb amputees. Research kinesiologists of the University of Washington collaborated with the Prosthetics Research Unit by conducting extensive investigation over a 5 year period of amputee running $(9,10)$.

It became evident that the state-of-the-art lower limb prostheses would have to be redesigned if real progress in amputee running was to be accomplished. No amount of muscle conditioning and training alone could be expected to accomplish major change in running performance. The prosthetic foot, which is the subject of this article, is an outgrowth of this amputee gait performance research (Figs. $1 A$ and $1 B$ ).

\section{DESIGN OF THE SEATTLE FOOT}

The VA Seattle foot conceptually provides storage of potential energy and is converted to 


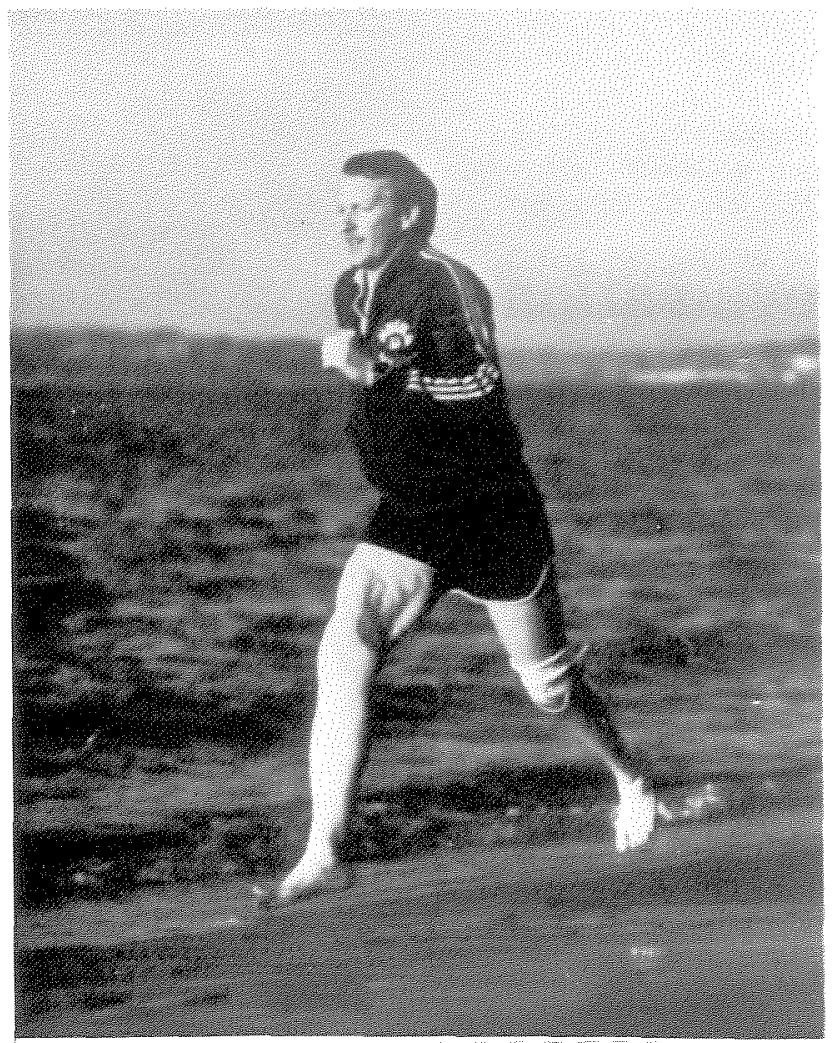

FIGURE 1 A

Below-knee amputee running barefoot with VA Seattle foot

kinetic energy throughout the weight-bearing phase of the gait cycle. This stored energy is progressively released as the foot continues through the toe-off phase to rebound and propel the body forward. In simple terms, weight deflects the keel through a predetermined range, then the keel "springs back" as weight is removed (Fig. 2).

Specifications were determined mathematically using information from the gait studies and transferring it to the bench testing of keel materials which could meet requirements $(1-3,8$, $11,12,15-18)$.

The first foot was fabricated from leaves of fiberglass combined with a light metal (4). When the foot was tried on patients, their response was one of remarkable acceptance. Continued use on test subjects brought out the problem areas: weight, breakage, individual amputee preferences for specific performance needs. After a series of refinements it became evident that the most satisfactory keel design would be monolithic. That is, with fewer component parts, the production cost would be lower, there would be less maintenance, and the response would be more uniform.

The present monolithic keel is composed of the acetal homopolymer, Dupont's Delrin ${ }^{\circledR}$. Its design form and physical performance are described in the engineering section of this article. Foaming was initially in the general shape of a foot and suitable for shoe fitting. This shape corresponded to the one ordinarily used for commercially available prosthetic feet. As the design progressed, we decided to prepare anatomical molds so that the foot would actually resemble a natural foot for those people who desired this type of cosmesis. A few amputees did not wish this natural appearance and accordingly were fitting with the standard and previously used blank-foot shape (Fig. 3).

These criteria were selected to guide development beyond the proof-of-concept fiberglass epoxy keels: 1) store and return energy (1-3/4 inch metatarsal deflection at 435 pounds vertical load);2) natural feel and stability; 3) useful life of 3 years; 4) lightweight ( 1 pound target); 5) reduced production costs.

An initial review of available space constraints within the foot shape, the large deflections required to simulate normal-foot A-P plane motions, and available material properties led to the following development approach: 1) uniform stress monolithic cantilever spring keel; 2) modular and compatible with existing prosthetic components (standard single-bolt attachment accessible from lower surface); 3) natural appearance; 4) minimize part count; 5) ultimate production keel to be molded; 6) fatigue: 50,00 cycles representing sprint running (load $=2.8 \times$ body weight) $-1,000,000$ cycles representing jogging (load $=1.4 \times$ body weight); $\gamma$ ) less than 0.06 inch permanent set at $3 \times$ body weight; 8 ) increase damping in the spring.

Early structural analysis of moldable materials showed that obtaining a sufficiently soft spring to allow required deflection at the forefoot was difficult, especially considering the demanding fatigue life requirements. Preliminary designs and structural analyses were conducted on nearly 


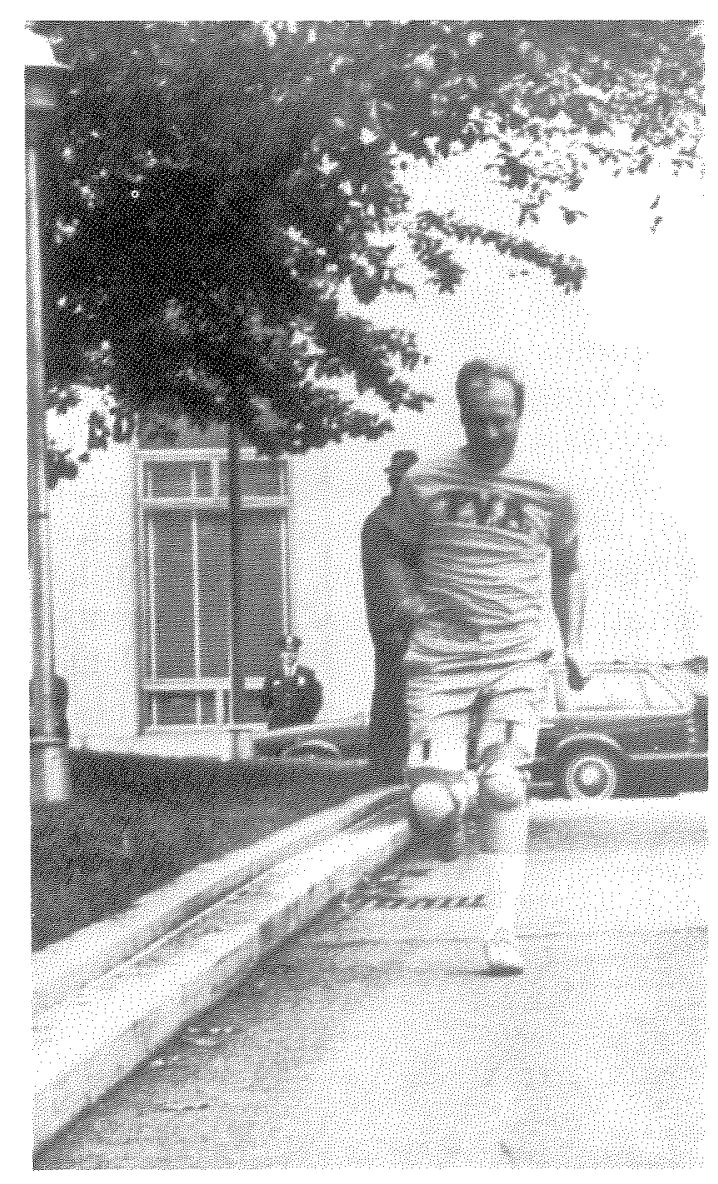

FIGUPR 1 P

Bilateral below-knee amputee running with VA Seattle foot

100 combinations of thermoset and themoplastic matrices and reinforcements. A constant width, parabolic tapered-depth beam was picked as the basic section for isotropic materials. Straight taper was used for oriented fiber composite candidates. A general keel shape consisting of a hook posterior to the bolt centerline and then descending to the metatarsals was selected because it would move the apparent "center of rotation" of the cantilever spring deflection as close as possible to the natural ankle center. It was also felt that increasing the outer extreme tension fiber length would improve fatigue life of the highly stressed keel materials. Load deflection testing of $1 / 4$ inch thick sections of the keel ("toes") was conducted early to guide design and analysis. Toe testing allowed quick evaluation of material and process combinations for which mechanical properties were not available. Over 50 of these representative sections were tested (Fig. 4).

When required load-deflection characteristics were obtained, a fatigue test was run on the candidate material toe section. The 2-cycle-persecond fatigue test device is shown in Figure 5. The test load was applied 23 degrees anterior to the shank centerline, simulating maximum dorsiflexion loads at heel-rise and toe-off.

of the several materials and shapes fatigue tested the Delrin 150 material (Dupont's acetal homopolymer) came closest to meeting all the criteria. Many materials which were strong enough were too stiff or had insufficient damping to allow a natural feel for the amputee. One of the shortcomings of the epoxy-fiberglass keel was that walking amputees felt "hurried" by the tooquick release of the stored spring energy. The significantly higher damping of Delrin ${ }^{\circledR}$ has eliminated this problem. Three-dimensional keels were then fatigue tested on the same device. A design was finally derived which met the fatigue criteria, although permanent set at the end of the conservative "straight-through" fatigue cycles was larger than desired ( 0.2 inch actual versus an objective of 0.06 inch). Subsequent patient testing on the natural intermittent loading cycle has not yet uncovered any creep problems.

\section{FIT PROTOCOL}

Clinical testing helped reveal flaws that have been corrected. Major problems areas were: 1) anterior keel tip ("toe") failures, which was corrected by pre-bending to a larger tip-up angle and thinning the section to reduce stress induced by the approximately 60 degrees toe deflection demanded during toe-off; 2) failures at the attachment bolt clearance hole in the bottom of the keel, corrected by providing additional reinforeing pad-up to carry loads around the hole; and 3) keel "punch-through" the foam on soft surface, e.g., barefoot in dry sand, corrected by adding a Kevlar fabric toe pad (Fig. 6).

Eight keel configurations are cumently required to cover the adult population of male shoe sizes 7 through 11 and body weights from 130 to 240 pounds. Use of the fit protocol shown in 
Figure 7 has resulted in high satisfaction for individuals with amputation.

Selection of a keel for an exceptionally high activity level might require the next stiffer keel to avoid breakage or to provide better feel (higher energy storage). Similarly a next softer keel might be prescribed for an inactive amputee. We have noted cases where an inactive patient (sometimes a bilateral amputee) has been fitted with a softer keel than body weight would dictate, and then found his activity level increasing to the point where he was able to break the keel. Since the keel is highly stressed during use by active amputees, all VA Seattle foot units are subjected to the rigorous acceptance test procedure presented in Figure 8.
Extensive acceptance clinical testing and evaluation was performed using 36 subjects (34 below-knee and 2 above-knee amputees). The high level of satisfaction/acceptance is recorded in Figures 9A, $B$, and $C$.

The foot was then placed into further evaluation with 500 volunteer amputees in 44 designated Veterans Administration Medical Centers across the United States. The feet were fitted to both new and currently worn prostheses by the subjects' prosthetists of choice as designated by the clinic prosthetic teams at participating stations. This study is being conducted by the Rehabilitation Research and Development Evaluation Unit under the direction of James B. Reswick, Sc.D., in collaboration with the Veter-
FIURE 2

How the foot works.

\section{FIGURE 3}

A: VA Seattle foot; $B$ : Simulated cosmetic foot (Otto Bock); $C$ : Standard SACH foot mold.
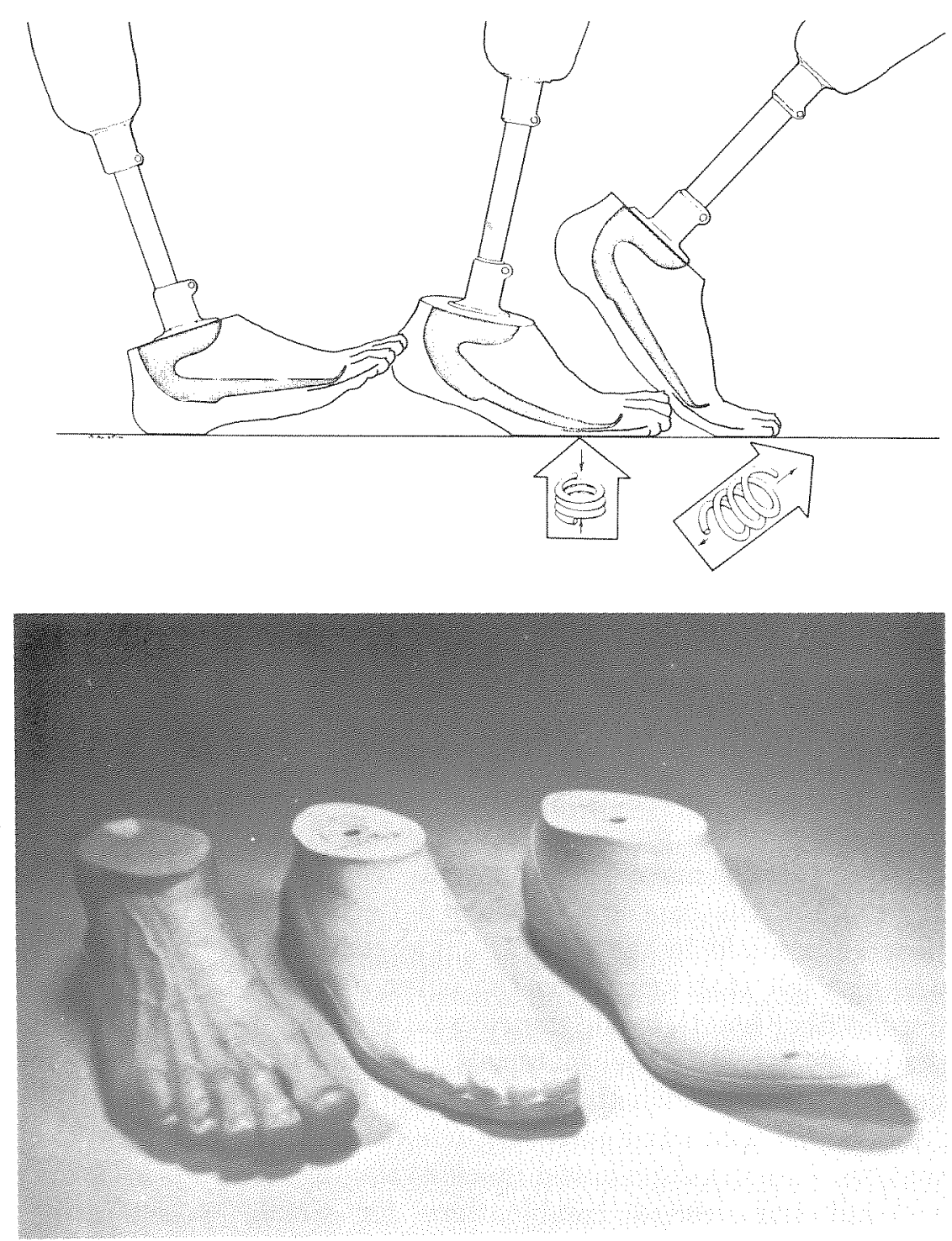


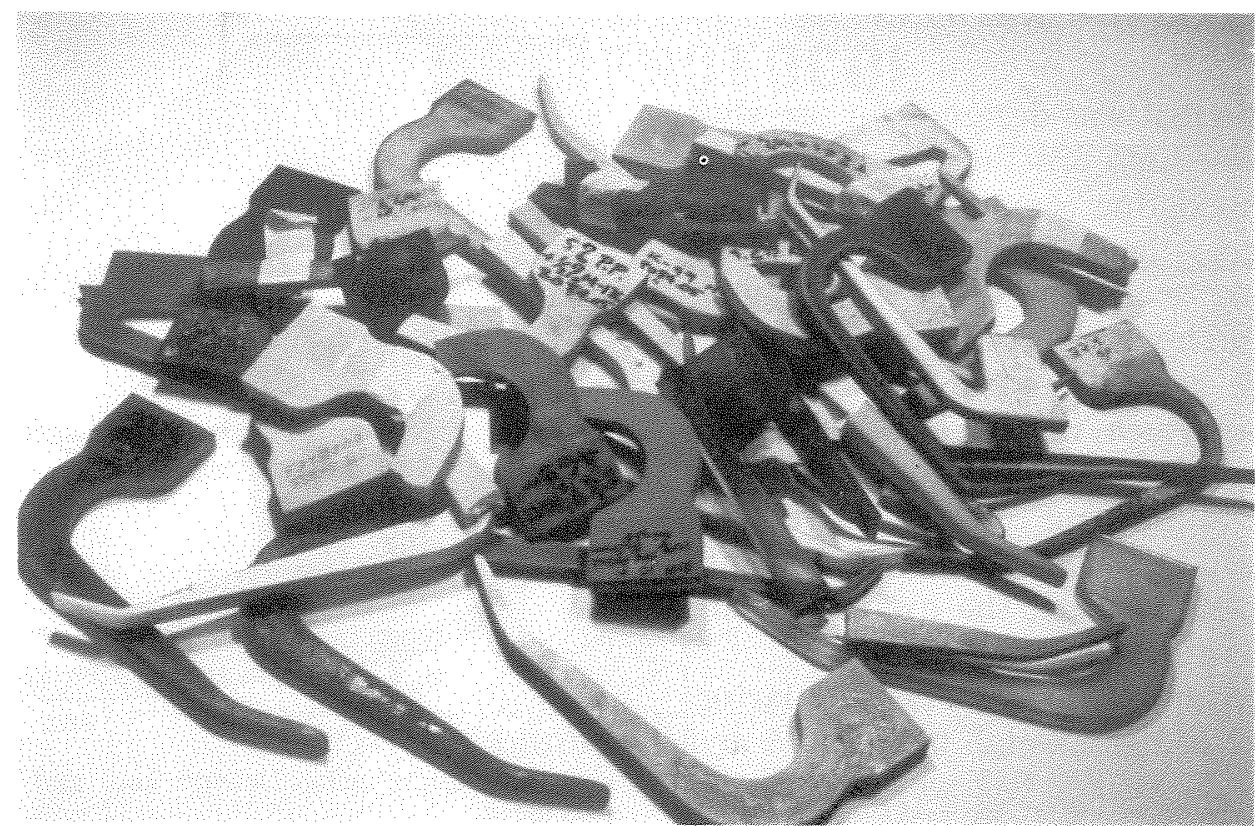

FIGURE

Some of the keel designs and materials evaluated.

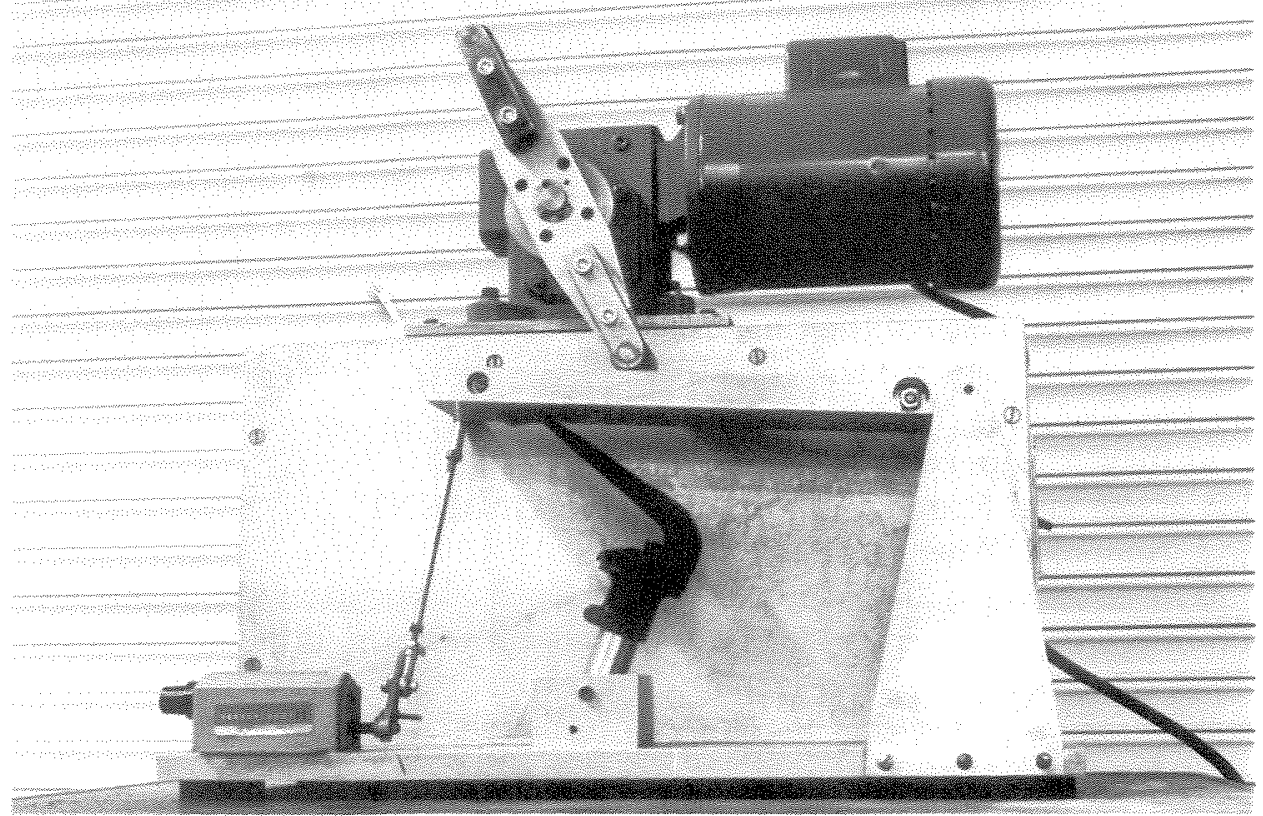

FIGURE 5

Bench-testing of keel for fatigue.

ans Administration Central Office's Prosthetics and Sensory Aids Service, which is directed by Frederick Downs, Jr. The Evaluation Unit was recently established by Margaret J. Giannini, M.D., as part of the Rehabilitation Research and Development Service. Data are now being collected and analyzed. Early unofficial reports indicate that they closely match the information derived from the small study.

A further commercial evaluation program was initiated and now completed using several hundred feet provided to prosthetists nationwide. This study, too, will be the subject of a subsequent report. 


\section{PROSTHETIC CONSIDERATIONS}

The VA Seattle foot was designed for use with conventional lower limb prostheses and is interchangeable with existing components. To accomplish this feature, the foot is attached to the prosthetic shank, endoskeletal or exoskeletal, with a single rigid bolt. Interchangeability, durability, simplicity, and cost were all addressed. Earlier designs, which involved a number of components, have been refined to the present monolithic keel. Minimizing components recluces servicing and cost. The VA Seattle foot has only three components: the keel, the external foam, and the toe reinforcement pad.

Weight of the appliance is a critical factor. Currently, prosthetic feet weigh between $1 / 2$ and $11 / 2$ pounds. The VA Seattle foot weighs approximately 1 pound, varying a small amount depending on the size used.

\section{PRESCRIPTION CRITERIA}

While the VA Seattle foot was initially designed for running, it can also be used for walking and is not necessarily contraindicated for lessactive people. Gait studies have shown that because the foot is flexible in the metatarsal area, it does not limit the forward travel of the tibia as it rotates over the foot, thus making the transition between foot-flat and toe-off smoother. By combining the ability to increase push-off through deflection of the keel, the foot improves both walking and running. When walking on uneven ground, the VA Seattle foot does not provide as much forefoot flexibility in the medial/ lateral plane as in the Greissinger or SAFE foot. Therefore, if the patient requires this motion, another foot should be selected. Continuing research is being directed to increase component compensation in additional planes as required by irregular surfaces, inclines, and steps. We desire to incorporate additional force-motion characteristics within the material rather than add or modify components.

As stated earlier, the VA Seattle foot can be used with success on athletic and less-active amputees, including individuals with bilateral amputation. The foot can also be used on all levels
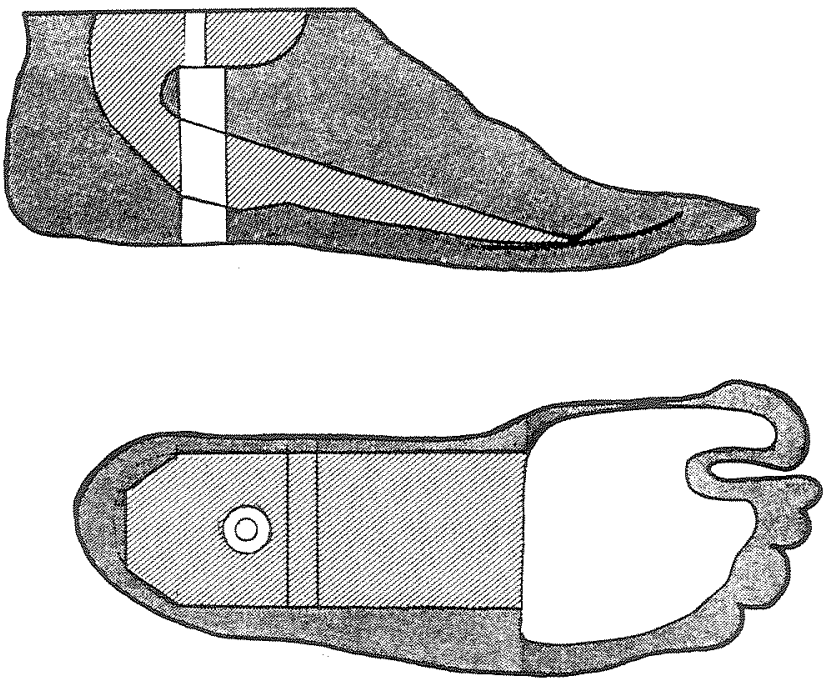

FIGURE 6

Current design using Delrin ${ }^{\text {B }}$ keel and Kevlar extension.

of lower-extremity amputation, with the exception of the Symes level. While the foot was designed to be interchangeable with existing prosthetic units, at the present time it cannot be used with a Hydra-Cadence, an R.O.L. rotator, or a Symes prosthesis.

The VA Seattle foot is designed to be worn with a shoe with a $3 / 4$ inch heel. If the patient wants to wear shoes with a lower heel, the foot should be wedged accordingly so that when it sits in the shoe, the top of the foot is parallel to the floor.

\section{ALIGNMENT}

Optimal performance of the VA Seattle foot is generally more difficult to achieve when it is attached to an existing prosthesis. Therefore it is recommended that the VA Seattle foot be realigned when applying it to a prosthesis. Generally speaking, the alignment of the VA Seattle foot is closer to that of the SAFE and Greissinger feet than the SACH foot. The amount of socket flexion or plantar-flexion differs considerably between the Seattle and SACH foot, as does the anterior and posterior position of the foot with respect to the socket.

As the foot is moved into plantar-flexion, the patient will be able to notice the level of push-off increase. However, as the level of push-off in- 


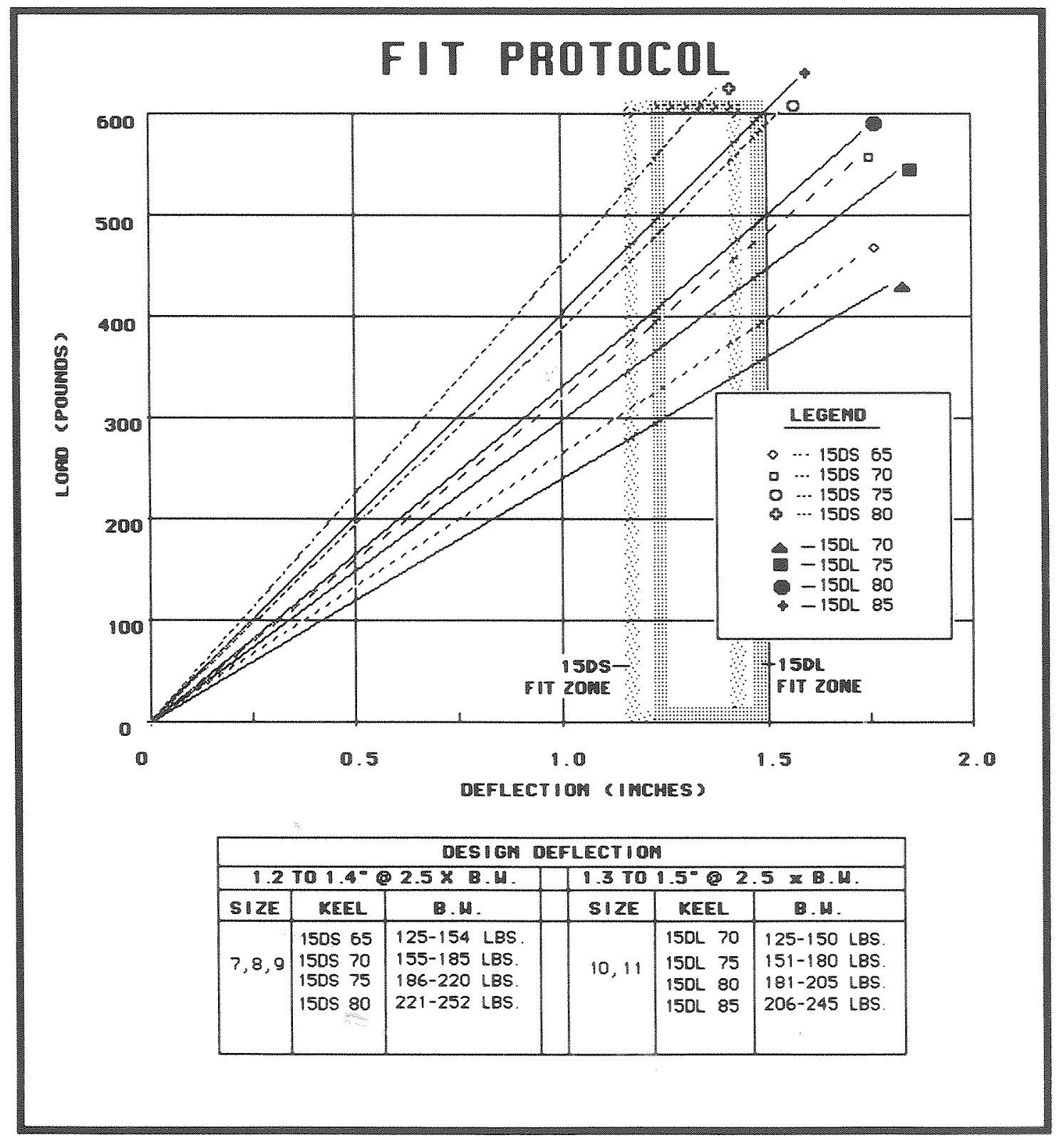

FIGURE 7

Fit protocol/load stroke chart.

creases, this increases the hyperextension moment of the knee during midstance and considerable effort needs to be taken to walk over the foot. In alignment, plantar-flexion must be balanced with knee hyperextension. The patient will also notice the amount of push-off or spring increase as the foot is moved posterior with respect to the socket. The prosthetist should find a compromise between the hyperextension moment at midstance and the level of push-off required. The knee should not be forced into hyperextension during any phase of gait. When aligning the foot for running, the prosthetist should externally rotate the foot 2 to 3 degrees beyond its normal position, since the prosthesis has a tendency to internally rotate while ruming. This compen. sates for the rotation so both feet are symmetrical.

When using the VA Seatle foot on above-knee or hip-disarticulation patient, standard multixis foot alignment procedures are used, except the pylon is placed in 2 to 3 degrees of posterior tilt. 


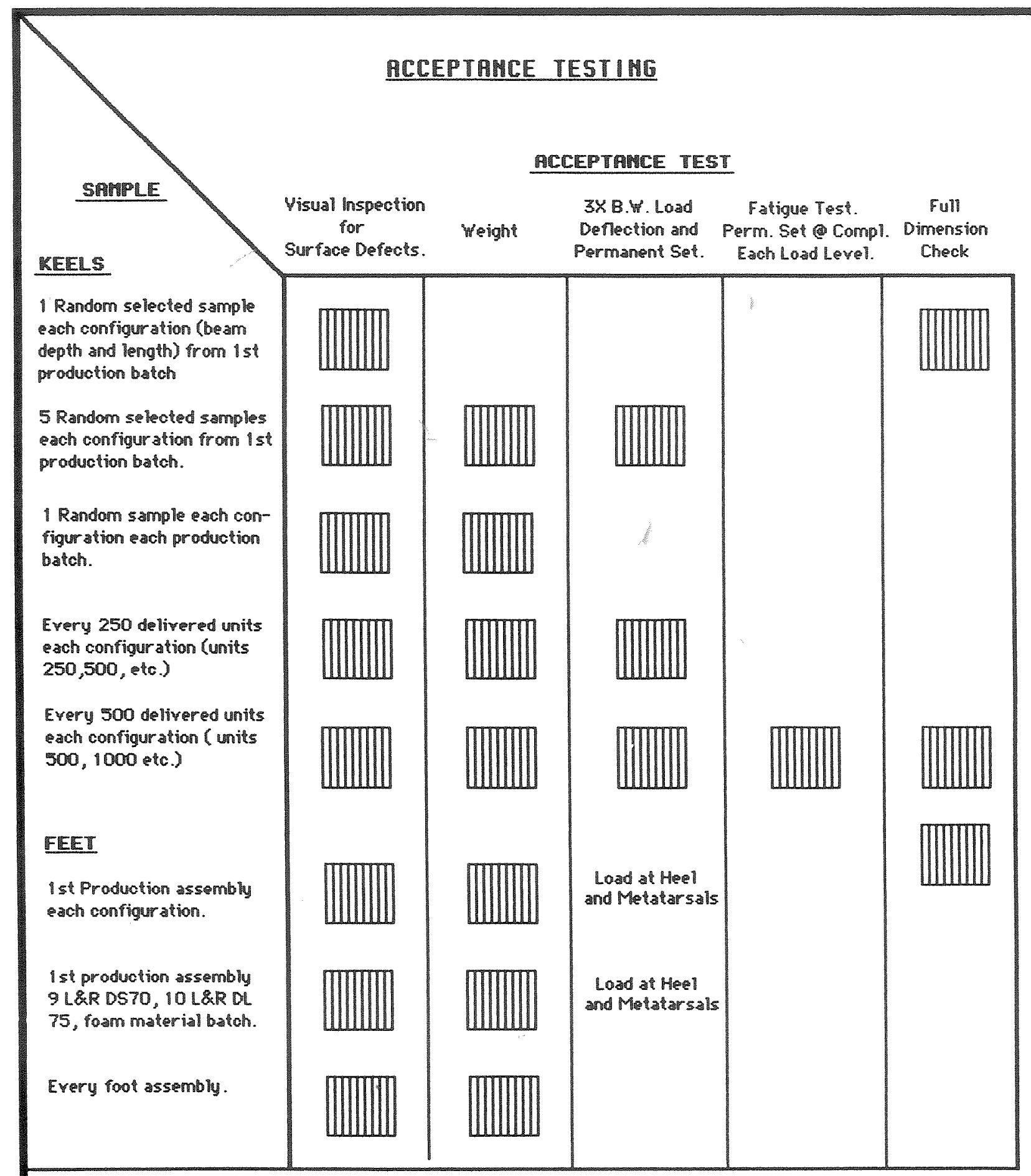

FORM MATEPIRL

Each now material batch: Measur fre foam density, cream and rise time, and shrinkage.

FIGURE 8

Acceptance test procedure. 

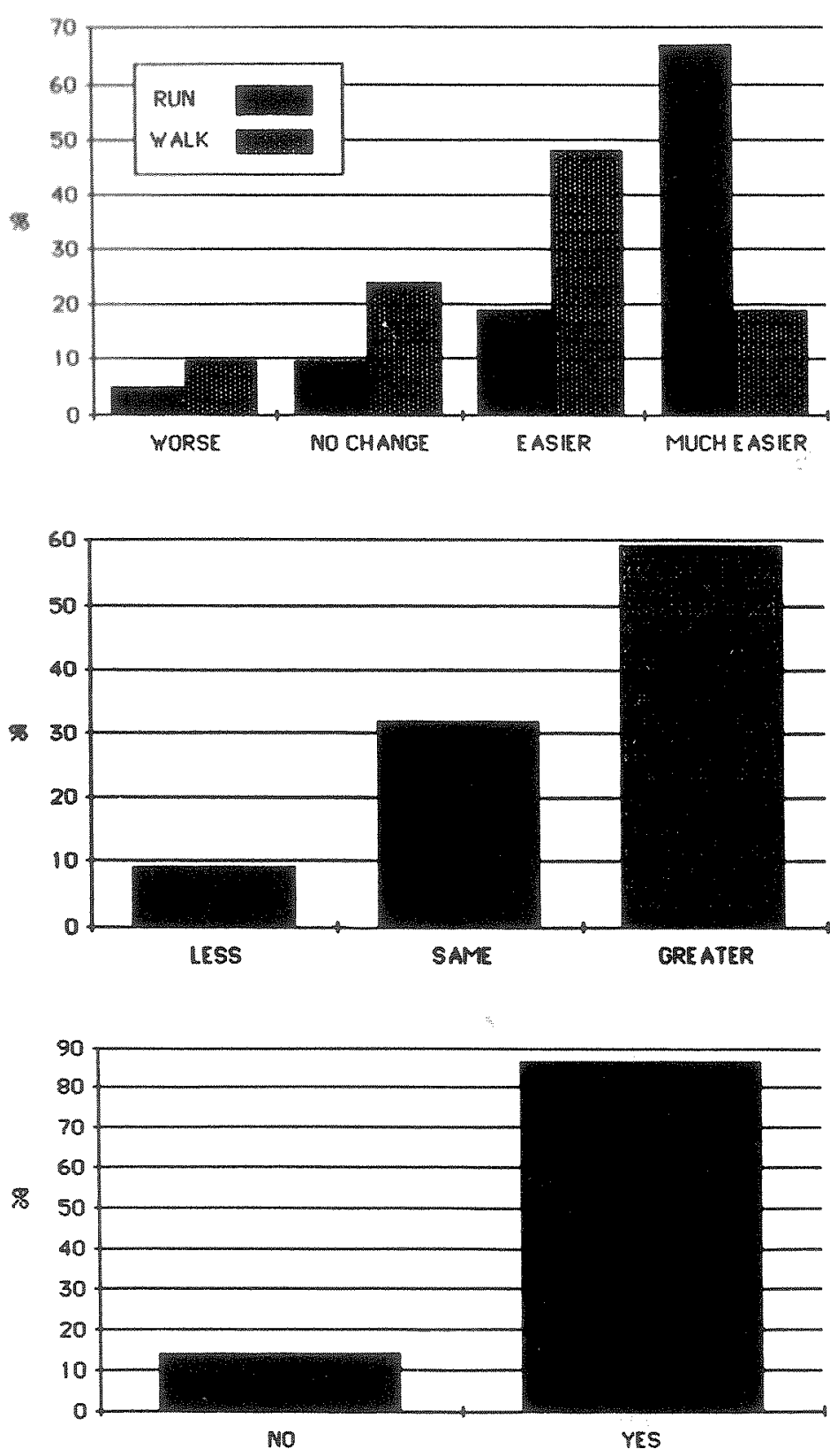

\section{FIGURE $9 A$}

Changes in walking versus changes in running with the VA Seattle foot: test subjects' responses to whether they had noticed any changes in these 2 activities.

\section{FIGURE 9P}

Endurance: test subjects' responses to whether they could perform their sports activities for less, same, or greater amounts with this foot design.

\section{FIGURE 9C}

Improved prosthesis: test subjects' responses to whether the Seattle foot was an overall improvement compared with their previous foot.
This preloads the keel and makes the pylon vertical during midstance. If the knee becomes unstable, the prosthetist can either increase the toe lever by plantar-flexing the foot or moving the knee center posterior to the TKA line.

\section{CLINICAL EVALUATION}

In the field of mobility aids, consumer acceptance is the final measure of success. This is especially so with prostheses. Satisfaction is necessarily relative since no prosthesis fully replaces function of the missing part. Functional and psychological loss are least evident in the congenital amputee whose experience does not include the missing member: Nonetheless, all amputees are fundamentally dissatisfied to some degree with the prosthesis. This dissatisfaction cannot be overcome since the prosthesis is ultimately measured in performance agamst the lost body part. However, when measured against a severely deformed, painful, infected or lifethreatening residual member, relief by amputa- 
Joumal of Rehabilitation Research and Development Vol. 22 No.3 July 1985

tion and subsequent prosthetic rehabilitation will prove a blessing. Nonetheless, the amputee must live with functional and body image alteration.

Comparisons between prostheses is another matter. Here, in most cases evaluation both subjective and objective provides a high degree of success measurement. Feedback data obtained by existing prosthetic users can be refined to provide an excellent engineering measurement tool Physical performance such as the ability to run at certain speeds and on varied surfaces, walking endurance, and measurements of metabolic activity also add to evaluation.

Our initial evaluation of the VA Seattle foot was carried out over a 3-year period with 36 amputees. All had previously worn a prosthesis. They were selected because of interest and desire to increase physical capabilities, primarily sports. Motivation was not a problem. None were paid for participating in the program. The experimental feet, and when necessary, prosthetic adjustment were furnished without cost to the test subjects. Personal interviews and questionnaires completed by prosthetists and amputees were summarized.

The amputee acceptance rate was high. When a failure occurred, test subjects often refused to give back the failed foot for study unless a replacement was provided so as to avoid returning to the use of their former appliance. The remarkable level of acceptance was paralleled by enhanced performance, greater endurance and in most instances, comfort.

\section{CONCLUSIONS}

A prosthetic foot is described, which consists of a monolithic composite keel engineered to store potential energy and release kinetic energy (force) during ground contact. Extensive clinical evaluation indicates high user acceptance. The present design is stabilized. Technology transfer for commercial availability has been carried out.

\section{ACKNOWLEDGMENTS}

The authors are indebted to the entire staff of Model \& Instrument Works Inc., especially Shirley C. Poggi for her anminstutive support, to Glen Austin and Ray Pye for then technow contribution, but in particular to Glen Camp-

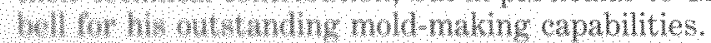

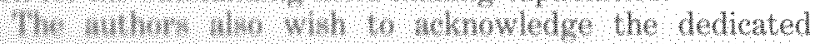

assistance provided to this project by Diane Lyons Harding, Research Secretary, Prosthetics Research Study.

They are grateful to the prosthetists, and patients who participated in the development and evaluation of the VA Seattle foot.

\section{RERERNCES}

1. AMERICAN ACADEMY OF ORTHOPAEDIC SURGEONS: Joint Motion: Method of Meaning and Recording. Chicago, IL: American Academy of Orthopaedic Surgeons, 1965.

2. ARMstrong RA: Skeletal muscle physiology. In: Sponts Medicine and Physology, Strauss RH (ed.). Philadelphia, PA: W.B. Saunders, 1979 , p. $29-48$.

3. BASMAJAN JV: Muscles Alie, Bnd ed. Baltimore, MD: Williams \& Wilkins, 1974.

4. Burgess EM, HitTenbERger DA, Forsgren SM, LINDH D: The Seattle prosthetic foot-a design for active sports: preliminary studies. Orthot Prosthet I 37 (1): $25-31,1983$.

5. EBERHART HD, InMAN VT, ETAL: Fundamental studies of human locomotion and other information relating to design of artificial limbs. Subcontractor's Report to the Committee on Artificial Limbs, National Research Council. Prosthetic Devices Research Project, Berkeley: College of Engineering, Univ of California, The Project, 1947. (Ser. CAL 5, 2 vols.)

6. ELFTMAN H: The basic pattern of human locomotion. Ann NY Acad Sci 51: 1207-1212, 1951.

7. INMAN VT: Conservation of energy in ambulation, Bull. Prosthet Res 10-9: 26-35, 1968.

8. ISPO-Standards for Lower-limb Prostheses. Report of a Conference, 1977.

9. Kegel B, CARPENTER M, Burgess EM: Funetional capabilities of lower-extremity amputees. Arch Phys Med Rehabil 59: 109-120, 1978.

10. Muler DI, PAsser MW, Burgess EM: Development and improvement of running skill in unilateral belowknee amputees. Final Report, VA Contract V663P-1254, bepartment of Kinesiology, University of Washington, 2 vols., 1984 .

11. MURMAY MP: Gat as a total pattern of movementincluding a biblography on gait. Am J Phys Med 6: 290 $333,1967$.

12. MURraY MP, Drought AB, Kory RC: Walking patterns of nomal men, I Bone I Surg 46A: 335-360, 1964.

13. PEIzER E, WRIGHT DW: Human locomotion. In: Prosthetic and Orthotic practice, Murdock G, ed. London: Edward Arnold, Ltd, 1969.

14. PEIzER E, WRIGHT DW, MAsON C: Human locomotion. Bull Prosthet Res 10(12): 48-105, 1969.

15. PERRY J: The mechanies of walking. A clinical interpretation. Phys Ther 47: 779-801, 1967.

16. RoBERTS TDM: Neurophysiology of Postural Mechanism. London: Butterworths, 1967.

17. SAUnders JB DE CM, INMAN VY, AND EBERHART HD: The major determinants in normal and pathological gait, J Bone Joint Surg 35A(3): 543-558, 1953.

18. SUTHERLAND DH: An electromyographic study of the plantar flexors of the ankle in normal walking on the level, J Bone Joint Surg 48A(1): 66-71, 1966.

19. WINTER DA: Energy assessment in pathological gait. Psysiother Can 30: 183-191, 1978. 